\title{
Guest Editors' Foreword
}

This issue of Discrete \& Computational Geometry contains the detailed proof by T. Hales and S. P. Ferguson of the Kepler conjecture that the densest packing of three-dimensional Euclidean space by equal spheres is attained by "cannonball" packing. This is a landmark result. This conjecture, formulated by Kepler in 1611, was stated in Hilbert's formulation of his 18th problem [8]. The proof consists of mathematical arguments and a massive computer verification of many inequalities.

In 1953 László Fejes Tóth [1] indicated the possibility of proving the Kepler conjecture by relating it to a nonlinear optimization problem over a compact set, and in 1964 he proposed a specific such inequality [2, pp. 295-299]. Such optimization problems can generically be termed "local density inequalities" as they involve some weighted measure of density associated to a neighborhood of each sphere center in a packing. Establishing a local density inequality yields a result stronger than the Kepler conjecture, since it requires the existence of a packing maximizing the local inequality simultaneously at every sphere center. Thus different local density inequalities are different mathematical results, although each may imply Kepler's conjecture as a corollary. Assuming that the optimization problem has a finite set of isolated global minima, and that the local minimality can be verified analytically in an open neighborhood of each of these minima, in principle the inequality can be proved by computer elsewhere using interval arithmetic. However, the size of the resulting optimization problems seemed far beyond the range of what could be solved by computer at that time, or even now, without new ideas to reduce the complexity of the problem.

An approach to prove the Kepler conjecture in this fashion was developed by Hales starting about 1992 (see [3] and [4]). A significant problem lies in formulating a local density inequality of this kind that is expected to be valid, and which represents an optimization problem small enough to be solvable in a reasonable time. A definite program to prove it was then initiated by Hales after 1995 in [5] and [6]. Further modifications of the local density inequality were required to obtain a tractable problem, and in this formulation process, important contributions were made by S. P. Ferguson, a student of Hales whose doctoral thesis work forms the paper Sphere Packing V in this issue. Significant innovations of Hales were needed to reduce the optimization problem to a tractable size. A first version of a complete proof was presented in six preprints, one joint with his 
Ph.D. student Ferguson, and one by Ferguson alone, posted on the mathematics arXiv in 1998.

A week-long workshop on the proof was given at the Institute for Advanced Study in January 1999. At that time the second editor wrote a summary of the top level of the proof tree, which was later included in [9]. The six papers were submitted to Annals of Mathematics and a team of reviewers was assembled by the first editor. The main portion of the reviewing took place in a seminar run at Eötvos University over a 3 year period. Some computer experiments were done in a detailed check. The nature of this proof, consisting in part of a large number of inequalities having little internal structure, and a complicated proof tree, makes it hard for humans to check every step reliably. Detailed checking of specific assertions found them to be essentially correct in every case tested. The reviewing process produced in the reviewers a strong degree of conviction of the essential correctness of this proof approach, and that the reduction method led to nonlinear programming problems of tractable size.

The second editor became involved in the reviewing process in January 2003. This resulted in a substantial revision and reorganization of the papers making clearer the top portion of the proof tree, and leading to a simplification of some definitions. The papers now include extra remarks motivating the choice of the given local density inequality, which is very complicated, and provide some reasons indicating why one should expect this particular local density inequality to hold. The revised version of the Ferguson paper now includes an explicit error bound away from the optimal density. An abridged version of the proof (excluding the paper of Ferguson) appears in Annals of Mathematics by Hales [7], with computer files and programs available on the Annals of Mathematics website. The detailed version of the proof, representing revised versions of the six 1998 preprints, appears in this special issue of Discrete \& Computational Geometry.

Summaries of some aspects of this proof and other local density approaches to the Kepler conjecture are discussed in the Seminar Bourbaki report of Oesterlé [10] and in [9].

The reviewing of these papers was a particularly enormous and daunting task. The first editor wishes to thank the following reviewers who substantially contributed to the process: Andras Bezdek, Michael Bleicher, Karoly Böröczky, Karoly Böröczky, Jr., Aladar Heppes, Wlodek Kuperberg, Endre Makai, Attila Por, Günter Rote, Istvan Talata, Bela Uhrin, and Zoltan Ujvary-Menyhard.

\section{References}

1. L. Fejes Tóth, Lagerungen in der Ebene auf der Kugel und im Raum, Springer-Verlag: Berlin, 1953. (Second edition 1972.) [See pp. 174-181.]

2. L. Fejes Tóth, Regular Figures, MacMillan: New York, 1964.

3. T. C. Hales, The sphere packing problem, J. Comput. Appl. Math. 44 (1992), 41-76.

4. T. C. Hales, Remarks on the density of sphere packings in three dimensions, Combinatorica 13(2) (1993), 181-197.

5. T. C. Hales, Sphere packings, I, Discrete Comput. Geom. 17 (1997), 1-51, eprint: math.MG/9811073.

6. T. C. Hales, Sphere packings, II, Discrete Comput. Geom. 18 (1997), 135-149, eprint: math.MG/9811074.

7. T. C. Hales, A proof of the Kepler conjecture, Ann. of Math. 162(3) (2005), 1065-1185. 
8. D. Hilbert, Mathematical problems, Bull.Amer. Math. Soc. 8 (1902), 437-479. Reprinted in: Mathematical Developments Arising from Hilbert Problems, Proc. Symp. Pure Math. XXVIII, American Mathematical Society: Providence, RI, 1976.

9. J. C. Lagarias, Local density bounds for sphere packings and the Kepler Conjecture, Discrete Comput. Geom. 27 (2002), 165-193.

10. J. Oesterlé, Densité maximale des empilements de sphères en dimension 3 [d'après Thomas $\mathrm{C}$. Hales et Samuel P. Ferguson], Séminaire Bourbaki, Exp. No. 863, Juin 1999.

Gábor Fejes Tóth

Hungarian Academy of Sciences

Jeffrey C. Lagarias

University of Michigan 